\title{
PHOTOVOLTAIC EFFECT ON PbTe $p-n$ JUNCTION IN THE PRESENCE OF MAGNETIC FIELD
}

\author{
Le Van Khoi, E. Grodzicka, B. Witkowska, T. Story \\ AND R.R. GALAzZKa \\ Institute of Physics, Polish Academy of Sciences \\ Al. Lotników 32/46, 02-668 Warszawa, Poland
}

\begin{abstract}
Photovoltaic spectra of PbTe $p-n$ junction have been measured in the infrared spectral region in the temperature range of $8-260 \mathrm{~K}$. The $p-n$ junctions have been formed by cadmium diffusion into the $p$-type PbTe crystals. From the positions of the photovoltaic maxima the energy gap of the diode material has been determined. In the presence of a magnetic field up to $7 \mathrm{~T}$, a pronounced oscillatory behavior of the photovoltage was observed in the Faraday and Voigt configurations. Experiments were performed as a function of the magnetic field intensity at a constant wavelength of the incident light. The energy of the interband magnetooptical transitions between the Landau levels in $\mathrm{PbTe}$ was determined and compared with the theoretical model of Adler, describing the energy band structure for the IV-VI compounds.
\end{abstract}

PACS numbers: 71.25.Tn, 78.20.Ls, 72.80.Ga

\section{Introduction}

Lead salt semiconductors have been known for their photoelectronic properties long before any study on the fundamental mechanisms involved was undertaken. The magnetooptical properties of this material group were studied by many authors [2-4]. In the majority of works the classical method of recording magnetoabsorption oscillations has been used. In this paper we report the observation of the oscillations of the photovoltaic effect in $\mathrm{PbTe} p-n$ junction prepared by diffusion of cadmium into the $p$-type PbTe crystals. The energies of the interband transitions between the Landau levels are determined and compared with the theoretical model [1]. 


\section{Experimental technique}

The diodes were fabricated from $\mathrm{PbTe}$ single crystals grown by the Bridgman method. The as-grown $p$-type crystals were cleaved along (100) surfaces and annealed by the two-zone technique in Te atmosphere in order to achieve a constant hole concentration of $3.5 \times 10^{18} \mathrm{~cm}^{-3}$ with a mobility about $1.3 \times 10^{5} \mathrm{~cm}^{2} /(\mathrm{V} \mathrm{s})$ at $T=4.2 \mathrm{~K}$. The $p-n$ junctions were prepared by diffusion of $\mathrm{Cd}$ into $p$-type substrates. The 30 min diffusion at $510^{\circ} \mathrm{C}$ produces a $p-n$ junction at depth about $2 \div 5 \mu \mathrm{m}$. The fabrication of the diode and scheme of the optical measurements have been described in detail elsewhere [5].

The measurements of photovoltage in the $p-n$ junction were performed in the Faraday configuration for both $\sigma^{+}$and $\sigma^{-}$circular polarizations of the incident light (for the unpolarized light as well) and in the Voigt configuration for linear polarization. In the absence of magnetic field the zero bias photovoltaic responses were measured. In the presence of magnetic field the photovoltages in the $p-n$ junction were measured at fixed photon energy by sweeping the magnetic field.

\section{Results and discussion}

We show in Fig. 1 the photovoltaic spectra of the $\mathrm{PbTe}$ Cd diode obtained at several temperatures. The shapes of these spectra are characteristic of all investigated diodes. We have related the energy gap of the diode material to the photon energy at which the photovoltaic response reaches its maximum. One can see in Fig. 1 that the maximum of the photoresponse at zero magnetic field shifts toward

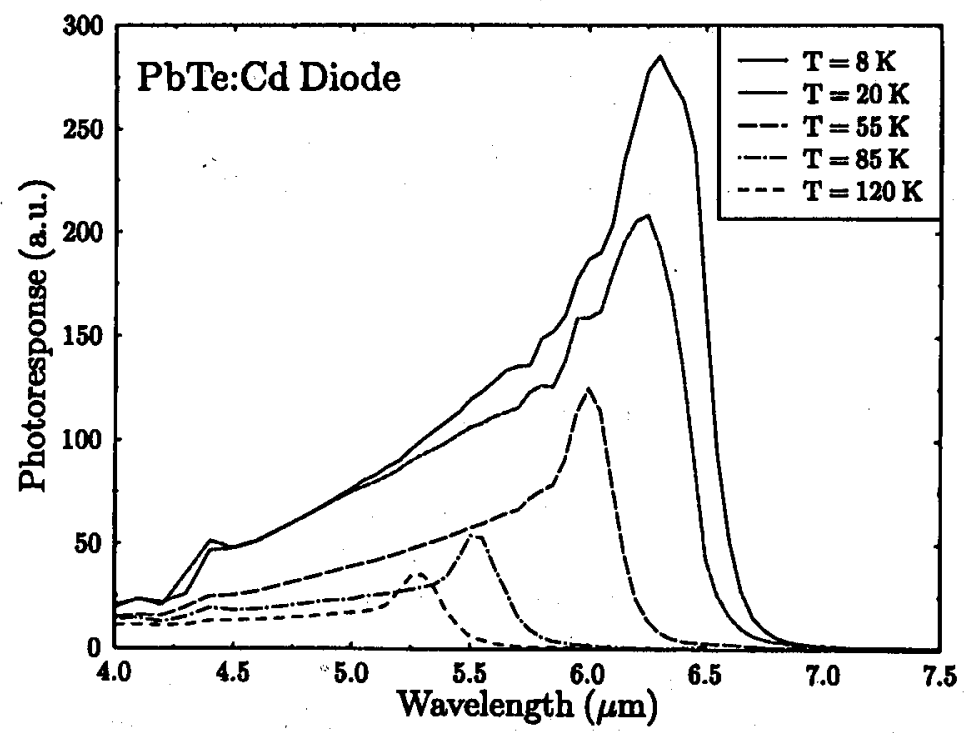

Fig. 1. Photovoltaic spectra of the PbTe:Cd diode. 


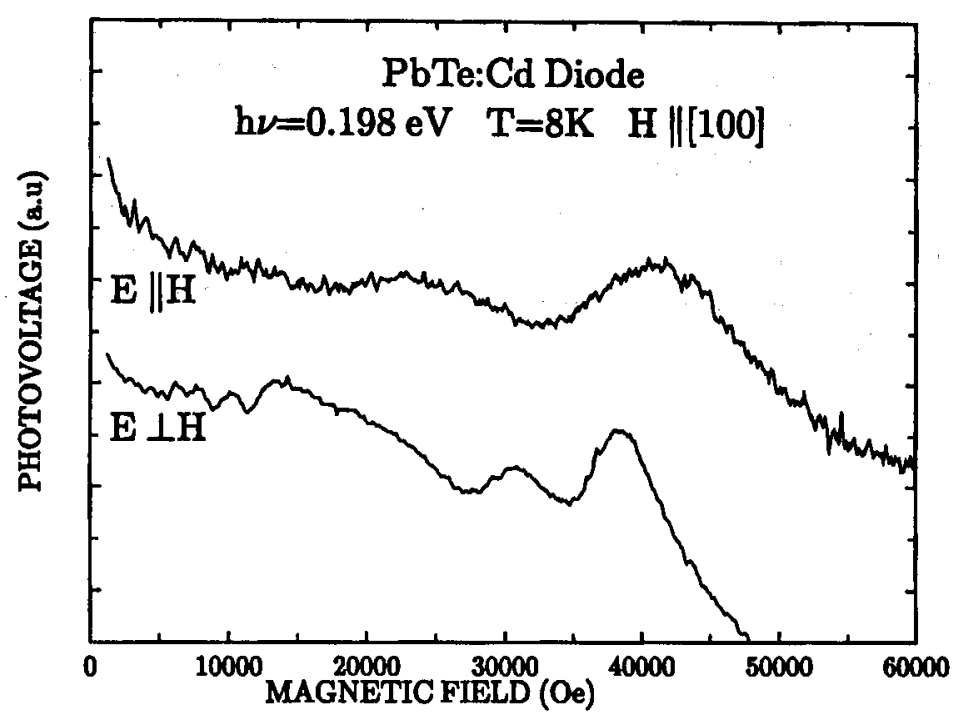

Fig. 2. Interband magnetooptical transitions in $\mathrm{PbTe} p-n$ junction in the Voigt configuration.

shorter wavelengths with increasing temperature. It indicates that the energy gap of $\mathrm{PbTe}$ increases with increasing temperature. This temperature dependence of the energy gap gives a coefficient between $4.0 \times 10^{-4}-4.2 \times 10^{-4} \mathrm{eV} / \mathrm{K}$. These data are in good agreement with the data in Ref. [2].

In Fig. 2 we show the zero bias photovoltage response of the $\mathrm{PbTe}$ :Cd diode recorded at photon energy $h \nu=0.198 \mathrm{eV}$ in the Voigt configuration (the magnetic field was along $\langle 100\rangle$ crystallographic axis). It can be seen that the photovoltage strongly oscillates in the magnetic field. For the investigated diodes with a thin diffusion layer and a high mobility of charge carriers, the photovoltage in $p-n$ junction reaches its maximum, when the interband magnetooptical transitions occur.

After the identification of the magnetooptical transitions, we have performed the fitting procedure for the experimentally determined transition energies with the energies calculated by the model of Adler [1] with the band parameters given in [3]. The fitting procedure shows that our experimental data are in good agreement with the theoretical model [1] for the transitions between the Landau levels with a quantum number $n \geq 1$ in the conduction and valence bands. But there is a considerable difference between the experimental and calculated energies within $\pm 2 \mathrm{meV}$ for the Landau levels with $n=0$ in the conduction and valence bands.

The observed deviation may be caused by the influence of the following effects: (1) the strong anisotropy of the surface of constant energy for $\mathrm{PbTe}$ $\left(P_{\mathrm{t}}^{2} / P_{1}^{2}=10.9\right) ;(2)$ the existence of the electric field located in $p-n$ junction. The more detailed estimation of the influence of those effects requires further study. 
724 Le Van Khoi, E. Grodzicka, B. Witkowska, T. Story, R.R. Gałqzka

\section{References}

[1] M.S. Adler, R.C. Hewes, D. Senturia, Phys. Rev. 7, 5186 (1973).

[2] U.I. Ravich, The Methods for Studying of Semiconductors in Use to PbTe, PbSe, $P b S$, Nauka, Moskva 1968 (in Russian).

[3] G. Bauer, H. Pascher, W. Zawadzki, Semicond. Sci. Technol. 7, 703 (1992).

[4] G. Bauer, H. Pasacher, M. Kriechbaum, Phys. Scr. Vol. T 19, 147 (1987).

[5] Le Van Khoi, Doctoral Thesis, Institute of Physics, Pol. Acad. Sci., Warszawa 1991 (unpublished). 